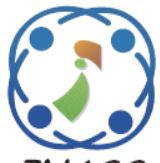

\title{
Cluster Based Routing Protocol Using Energy Centric Multi Objective Salp Swarm Algorithm for WSNs
}

\author{
Gundeboyina Srinivasalu ${ }^{1 *}$ \\ Hanumanthappa Umadevi \\ ${ }^{I}$ Department of Electronics \& Communication Engineering, Cambridge Institute of Technology, Bengaluru, India \\ ${ }^{2}$ Department of Electronics \& Communication Engineering, \\ Dr. Ambedkar Institute of Technology, Bengaluru, India \\ * Corresponding author's Email: Srinivasalu.ece@cambridge.edu.in
}

\begin{abstract}
Wireless Sensor Network (WSN) is an immense collection of low-power, intelligent and multifunctional sensor nodes for sensing and monitoring the environmental conditions. The information collected from sensors are transmitted to the sink or Base Station (BS). The sensors in the WSN use the battery energy and the energy consumption of the nodes are considered as an important constraint in the network. In order to overcome the issue related to the energy consumption, a cluster based routing protocol is developed in the WSN. In this paper, an appropriate Cluster Head $(\mathrm{CH})$ selection and route generation are obtained using the Energy Centric Multi Objective Salp Swarm Algorithm (ECMOSSA). The main objective of using ECMOSSA is to improve the network lifetime of the WSN by minimizing the node's energy consumption. The performance of the proposed ECMOSSA method is evaluated by means of alive nodes, total energy consumption, total packets received by the BS, throughput and network lifetime. Moreover, the ECMOSSA method is evaluated with one classical approach namely Low-Energy Adaptive Clustering Hierarchy (LEACH) protocol as well as this ECMOSSA is compared with Grey Wolf Optimizer (GWO)Dual Hop Routing (DHR) method and Cat- Salp Swarm Algorithm (C-SSA) to evaluate the efficiency of ECMOSSA. The last node dies (i.e., network lifetime) of the ECMOSSA is 1704 that is high when compared to both the LEACH and GWO-DHR method.
\end{abstract}

Keywords: Cluster head selection, Energy centric multi objective salp swarm algorithm, Energy consumption, Network lifetime, Route generation, Wireless sensor network.

\section{Introduction}

WSN is a type of self-organizing networks that composes of numerous sensor nodes to process the sensing and communication tasks using the radio waves. The purpose of the WSN is to identify the mobile target's movement (e.g. fire spread and wildlife) or observe the conditions (e.g. humidity and temperature) $[1,2]$. The sensor nodes in the WSN senses various parameters such as moisture level, temperature, light intensity, vibration, pressure and so on [3]. This WSN plays a major role in the abandoned real time applications such as Industrial monitoring, surveillance in battle field, climatic and weather monitoring, health monitoring, natural disaster prevention, traffic monitoring, environmental condition monitoring and so on [4].
Sensors in WSN have many advantages such as selfidentification, time awareness, self-diagnosis and simple installation while coordinating with the remaining sensors to generate the dynamic selforganized networks. However, these sensors have different constraints such as restricted energy, memory and computational ability $[5,6]$.

Generally, the WSN is densely installed in the harmful places where the recharging or replacement of the battery is impossible as well as the human monitoring scheme is very risky [7]. The communication task carried out by the sensor node consumes a high amount of energy when compared to remaining tasks such as sensing and processing tasks [8]. Accordingly, the energy efficiency is considered as the main constraint because of the restricted battery capacity of the sensors [9]. In order 
to overcome those issues, the clustering and routing are used due to its energy efficiency and reliability in data transmission [10]. In clustering, the sensors are divided into different clusters and each cluster in the network has one $\mathrm{CH}$ whereas the remaining sensors are cluster members $[11,12]$. The $\mathrm{CHs}$ selected from the network has important tasks such as coordinating sensor nodes, data aggregation and data transmission between the other $\mathrm{CH}$ [13]. Thus, the clustering helps to improve the throughput and network lifetime by minimizing the long distance communications [14]. Additionally, the routing algorithm is developed in the clustered WSN to guide the $\mathrm{CH}$ selection and identify the optimal routing path to reduce the node's energy consumption [15].

In [16], the Salp Swarm Algorithm (SSA) is already used for accomplishing the $\mathrm{CH}$ selection. However, in depth it fails to analyse its performances in the routing path generation. Moreover, this SSA based $\mathrm{CH}$ selection is only used to overcome the nonuniform load assignment and energy hole issues. Furthermore, the major contributions of this research using ECMOSSA are given as follows:

- In ECMOSSA, the selection of $\mathrm{CH}$ is carried out using five different fitness function values such as residual energy, intra cluster distance, distance from the $\mathrm{CH}$ to $\mathrm{BS}$, node degree and node centrality.

- Next, the routing path generation using ECMOSSA is obtained by considering three different fitness function values such as residual energy of the $\mathrm{CH}$, the distance between the $\mathrm{CH}$ and $\mathrm{BS}$ and node degree.

- Therefore, an adequate $\mathrm{CH}$ selection and routing path generation are used to decrease the node's energy consumption that results in a better network lifetime.

The overall organization of the paper is given as follows: Section 2 provides the literature survey about the recent techniques related to the cluster based routing in WSN. The problem statement identified from the literature survey and solutions given by the ECMOSSA is stated in Section 3. The preliminaries considered for the ECMOSSA is described in Section 4. Section 5 describes the ECMOSSA based $\mathrm{CH}$ and routing path selection. Next, the results and discussion for the ECMOSSA is clearly detailed in Section 6. Finally, the conclusion is made in Section 7.

\section{Literature survey}

The literature survey of the existing techniques associated to the clustering and routing methods in the WSN are described in this section.
Lalwani [17] presented Optics Inspired Optimization (OIO) to create the cluster and route in the WSN. The $\mathrm{CH}$ selection in OIO was considered various factors such as node degree, energy and distance. The next hop $\mathrm{CH}$ with high residual energy was chosen to avoid the packet loss among the network. But, the OIO based clustering and routing was affected according to the network size.

Mittal [18] developed the $\mathrm{CH}$ and route selection among the nodes using the GA algorithm. The Threshold-sensitive Energy-efficient Routing Protocol (TERP) algorithm was used for transmitting the data in the cluster. The multi-hop communication was accomplished by GA and it was used to improve the load balancing and to decrease the energy consumption of the distant $\mathrm{CHs}$. But the $\mathrm{CH}$ reselection process mainly depended on the remaining energy of the nodes.

Lalwani [19] presented the Biogeography Based Optimization (BBO) for clustering by considering various objective functions such as residual energy, intra-cluster distance and distance between $\mathrm{CHs}$ and the $\mathrm{BS}$ for better $\mathrm{CH}$ selection. Then the node degree, residual energy and Euclidean distance were considered in route generation of BBO. The packets collected by the BS is increased by minimizing node's energy. However, the residual energy of the network was slightly degraded when the BS is located outside the network.

Daneshvar [20] presented the Grey Wolf Optimizer (GWO) to choose the CHs in the network. In the $\mathrm{CH}$ selection, the solution was evaluated using the current residual energy of each node and predicted energy consumption. Next, a Dual Hop Routing (DHR) was used to transfer the data packets through the network. Further, the GWO algorithm was used to eliminate the unwanted execution of clustering to minimize the energy waste. However, the GWO algorithm doesn't consider the distance factor while selecting the $\mathrm{CHs}$ from the network.

Morsy [21] developed the hybrid Particle Swarm Optimization (PSO) and Gravitational Search Algorithm (GSA) to select the $\mathrm{CH}$ from the nodes. The fitness function values considered in the $\mathrm{CH}$ selection were distance to BS, energy efficiency and intra cluster distance. Here, the multi hop communication between the selected CHS were detected using the cost function that composes of the $\mathrm{CH}$ 's residual energy, distance between the $\mathrm{CHs}$ and distance among the $\mathrm{CH}$ and BS. Thus, the optimal selection of $\mathrm{CH}$ was used to balance the energy consumption. However, this hybrid PSO-GSA algorithm failed to analyze the last node die.

Vinitha, Rukmini, and Sunehra [22] developed a Cat-SSA (C-SSA) algorithm based energy efficient 
routing to select appropriate hops during the route generation. At first, the Low-Energy Adaptive Clustering Hierarchy (LEACH) protocol was developed to select the $\mathrm{CH}$ in the network that used to decrease the traffic in the network. Next, the CSSA was used to select an appropriate hop based on the energy constraints. However, the random $\mathrm{CH}$ selection property of LEACH created the higher energy consumption.

\section{Problem statement}

The problems found from the literature survey and solutions given by the proposed ECMOSSA method are stated in this section.

Generally, the performances of WSN are mainly affected due to the inappropriate selection of fitness function parameters. For example, the reselection of the $\mathrm{CH}$ in GA [18] is mainly depends on the residual energy. Additionally, the GWO algorithm [20] is selected the $\mathrm{CH}$ using the residual energy of a node and predicted energy consumption. Both GA [18] and GWO [20] are failed to consider the energy while selecting the $\mathrm{CH}$. This results in higher energy consumption during the data transmission. Moreover, the node's residual energy is slightly degraded, when the BS is placed outside the network.

\section{Solution:}

The problems related to the inappropriate selection of fitness function parameters is avoided by considering an adequate fitness function parameter to select the $\mathrm{CH}$. In the $\mathrm{CH}$ selection process, there are five fitness values such as residual energy, intra cluster distance and distance from the $\mathrm{CH}$ to $\mathrm{BS}$, node centrality and node degree. The aforementioned fitness parameters are employed to choose an adequate $\mathrm{CH}$ during the data transmission. Additionally, the residual energy of the $\mathrm{CH}$, distance between the $\mathrm{CH}$ and $\mathrm{BS}$ and node degree are considered to select the optimal shortest path to transmit the data packets. Hence, this optimal $\mathrm{CH}$ and route selection using ECMOSSA are helps to improve the network lifetime while obtaining a high amount of data packet transmission to the BS.

\section{System model}

This section delivers the information about the network model and energy model considered in this ECMOSSA method.

\subsection{Network model}

The network model of ECMOSSA method composes of BS and $N$ amount of sensors which are randomly positioned in the network area. A huge amount of sensors is used to accomplish the network connectivity. The assumptions considered in this network model are given as follows:

1. The BS and sensor nodes remain static, once it is deployed in the network area.

2. Sensor nodes have the capacity to report their position to the BS and other nodes.

3. All sensors in the WSN have initial energy.

4. The distance is calculated using Euclidean distance which is expressed in the Eq. (1).

$$
d=\sqrt{\left(x_{i}-x_{j}\right)^{2}-\left(y_{i}-y_{j}\right)^{2}}
$$

where, $x_{i}, y_{i}$ and $x_{j}, y_{j}$ are the coordinates of the $i^{t h}$ and $j^{\text {th }}$ node.

5. In execution process, all the sensors transmit the fitness function values to the BS. Next, the $\mathrm{BS}$ selects the $\mathrm{CH}$ using the fitness function i.e., $M$ amount of $\mathrm{CH}$ is detected from the $N$ amount of sensor nodes. Accordingly, the non $\mathrm{CHs}$ are assigned to the respective $\mathrm{CH}$. Finally, the data packets are transmitted via the $\mathrm{CHs}$ using the route acquired from routing algorithm.

\subsection{Energy model}

In ECMOSSA method, the 1st order radio model is used to define the transmitting and receiving energy of the sensors. In this energy model, either free space (i.e., $d^{2}$ power loss) or multipath fading (i.e., $d^{4}$ power loss) channel model is considered based distance $(d)$ among the source and destination. The free space model is utilized, when the distance is lesser than the distance threshold $\left(d_{0}\right)$. Otherwise, the multipath fading model is used during energy calculation. Eq. (2) is used to calculate the energy depletion of the node while transferring $l$ bit data packets over the distance $(d)$.

$$
\begin{gathered}
E_{T X}(l, d)= \\
\left\{\begin{array}{c}
l \times E_{\text {elec }}+l \times E_{f s} \times d^{2} \quad \text { if } d<d_{0} \\
l \times E_{\text {elec }}+l \times E_{m p} \times d^{4} \quad \text { if } d \geq d_{0}
\end{array}\right.
\end{gathered}
$$

where, the energy dissipated by the electronic circuit per bit is represented as $E_{\text {elec }}$; the free space and multipath coefficient factors are represented as $E_{f s}$ and $E_{m p}$ respectively. Then the distance threshold 
used to specify the free space and multipath fading is calculated using Eq. (3).

$$
d_{0}=\sqrt{\frac{E_{f s}}{E_{m p}}}
$$

Eq. (4) shows the receiving energy calculation for $l$ bit data packets.

$$
E_{R X}(l, d)=l \times E_{\text {elec }}
$$

\section{ECMOSSA method}

The proposed ECMOSSA method has three main phases such as $\mathrm{CH}$ selection, cluster formation and generation of routing path. From the network, an optimal CHs are selected using the residual energy, intra cluster distance and distance from the $\mathrm{CH}$ to $\mathrm{BS}$, node degree and node centrality. Additionally, an appropriate routing path is detected by considering the residual energy of the $\mathrm{CH}$, distance between the $\mathrm{CH}$ and $\mathrm{BS}$ and node degree. The main objective of this ECMOSSA method is to design an energy efficient WSN to improve the network lifetime. The flowchart of the ECMOSSA method is shown in the Fig. 1.

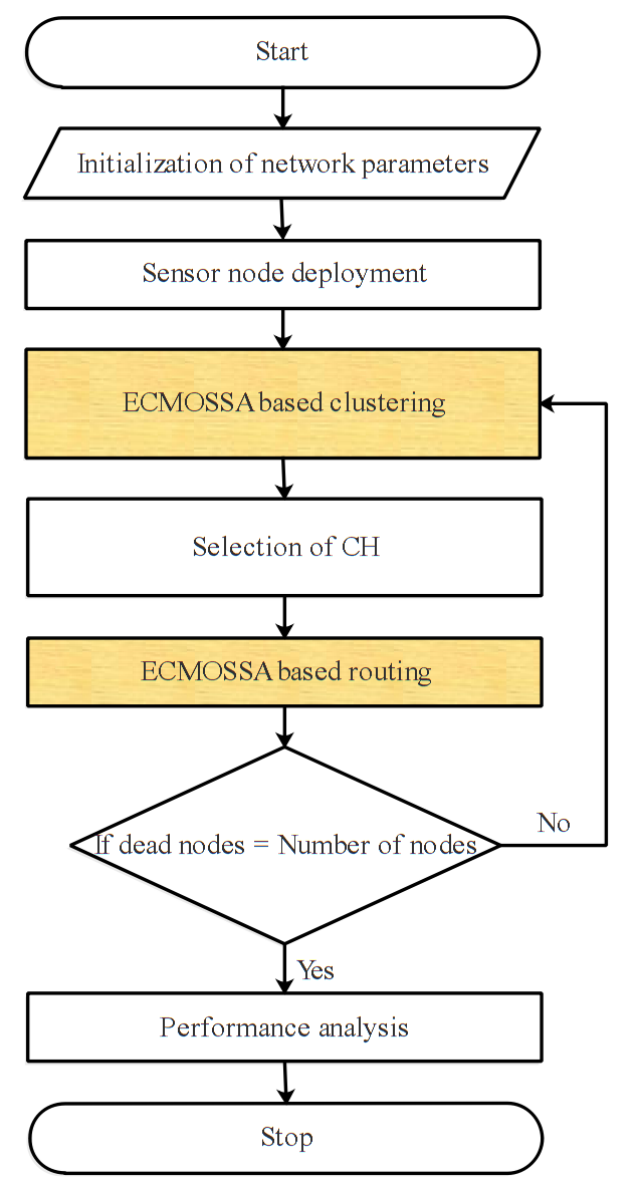

Figure. 1 Flowchart of the ECMOSSA method

\subsection{Overview of the salp swarm algorithm}

SSA is developed by the Mirjalili [23] at 2017 and this SSA is inspired by the behaviour of the salps. Generally, the salps are belongs to the Salpidae family which has transparent barrel-shaped body. The tissues in the SSA are identical to the jelly fishes and it moved in which the water is pumped over the body has a propulsion to move forward.

The population of the SSA separates into two types such as leader and followers to obtain the mathematical model of the salp chain. The salp present in the front of the salp chain is termed as leader whereas the other salps in the chain is termed as followers. The salp term represents that the leader directs the swarm and the followers in the SSA.

The salp position is represented in the $n$ dimensional search space, where the amount of variables in the SSA is represented as $n$. Hence, the salp location is saved at 2 dimensional matrix called $x$. The salp's food source in the search space is $F$ that is considered as a target of the swarm. The location updates the salp leader of the SSA is obtained using the Eq. (5).

$$
\begin{gathered}
x_{j}^{1}= \\
\begin{cases}F_{j}+c_{1}\left(\left(u b_{j}-l b_{j}\right) c_{2}+l b_{j}\right) & c_{3} \geq 0 \\
F_{j}-c_{1}\left(\left(u b_{j}-l b_{j}\right) c_{2}+l b_{j}\right) & c_{3}<0\end{cases}
\end{gathered}
$$

where, the $1^{\text {st }}$ salp position at dimension $j$ is represented as $x_{j}^{1}$; the source of food at dimension $j$ is represented as $F_{j}$; the $j^{\text {th }}$ dimension's upper and lower bound are represented as $u b_{j}$ and $l b_{j}$ respectively; $c_{1}, c_{2}$ and $c_{3}$ are the random numbers.

Eq. (5) shows that the leader updates the location according to the food source. In SSA, the coefficient $c_{1}$ i.e., expressed in Eq. (6) is a key parameter as it balances the exploitation and exploration behavior of salp.

$$
c_{1}=2 e^{-\left(\frac{4 r}{r_{\max }}\right)^{2}}
$$

where, the $r$ and $r_{\max }$ represents the current iteration and maximum number of iterations respectively. Moreover, the parameters of $c_{2}$ and $c_{3}$ are random numbers that is uniformly generated in the range of $[0,1]$. These parameters are used to define the next location at the dimension $j$ that shows the position moves towards positive infinity or negative infinity.

The following Eq. (7) i.e., Newton's law of motion is used for updating the follower's location. 


$$
x_{j}^{i}=\frac{1}{2} a t^{2}+v_{0} t
$$

where, $i \geq 2$, the location of the follower $i$ at dimension $j$ is represented as $x_{j}^{i}$; time is $t$; initial speed is $v_{0}, a=\frac{v_{\text {final }}}{v_{0}}$ and $v=\frac{x-x_{0}}{t}$. In this optimization algorithm, the time is iteration and difference among the iteration is 1 . Consider $v_{o}=0$, then the Eq. (7) is rewritten as shown in the Eq. (8).

$$
x_{j}^{i}=\frac{1}{2}\left(x_{j}^{i}+x_{j}^{i-1}\right)
$$

where, $i \geq 2$, the location of the follower $i$ at dimension $j$ is represented as $x_{j}^{i}$. Hence, the salp chain is simulated using the Eq. (5) and (8). Here, this SSA is modified into ECMOSSA used to select the $\mathrm{CH}$ and generate the routing path over the network.

\subsection{CH selection using ECMOSSA}

In this phase, the optimal sensors are chosen as $\mathrm{CH}$ using the residual energy, intra cluster distance and distance from the $\mathrm{CH}$ to $\mathrm{BS}$, node degree and node centrality.

\subsubsection{Salp representation}

The potential solution is termed as salp in the ECMOSSA and these salp represents the sensors to be selected as the $\mathrm{CHs}$ during $\mathrm{CH}$ selection phase. Each salp's dimension is equal to the amount of $\mathrm{CHs}$ which are required to be selected as $\mathrm{CHs}$ through the network.

\subsubsection{Salp initialization}

In salp initialization process, each salp location is initiated with a random ID of node among the 1 and $N$. Consider the $i^{\text {th }}$ salp i.e., $x^{i}=x_{1}^{i}, x_{2}^{i}, \ldots, x_{j}^{i}$, where dimension is represented as $j$ that is equivalent to the number of $\mathrm{CHs}$, where the location of an each salp is $x_{j}^{i}, 1 \leq a \leq j$ which represents the node ID among the 1 and $N$.

\subsubsection{Fitness function formulation for ECMOSSA based $\mathrm{CH}$ selection}

The fitness function used in the ECMOSSA to select the $\mathrm{CH}$ is formulated as follows.

\subsubsection{Residual energy}

The $\mathrm{CH}$ collects the data from its cluster members and aggregates the received data as well as the data is sent to the BS. To accomplish the aforementioned tasks, the $\mathrm{CH}$ should have higher energy than the cluster members. Hence, the sensor with high energy is desired highly during $\mathrm{CH}$ selection. The sensor's residual energy $\left(f_{1}\right)$ considered in the fitness function is expressed in Eq. (9).

$$
f_{1}=\sum_{i=1}^{M} \frac{1}{E_{C H_{i}}}
$$

where, the residual energy of the $\mathrm{CH}$ in the network is represented as $E_{\mathrm{CH}_{i}}$.

\subsubsection{Intra cluster distance}

The intra-cluster distance represents the average distance among the $\mathrm{CH}$ and its non- $\mathrm{CH}$ nodes. The distance specified in the section 3.2 is used to define the sensor's energy consumption. Energy consumption of the sensor is less, when the intra cluster distance for the respective $\mathrm{CH}$ is less. Eq. (10) expresses the 2 nd objective function i.e., intra-cluster distance $\left(f_{2}\right)$.

$$
f_{2}=\sum_{j=1}^{M}\left(\sum_{i=1}^{I_{j}} \operatorname{dis}\left(N_{i}, C H_{j}\right) / I_{j}\right)
$$

where, the distance between the $i^{\text {th }}$ sensor to the $j^{\text {th }} \mathrm{CH}$ is represented as $\operatorname{dis}\left(\mathrm{N}_{i}, \mathrm{CH}_{j}\right)$ and the intra cluster members for the respective $\mathrm{CH}$ is represented as $I_{j}$.

\subsubsection{Distance from $\mathrm{CH}$ to $\mathrm{BS}$}

The distance among the $\mathrm{CH}$ and destination (BS) is considered as 3rd objective for the $\mathrm{CH}$ selection. Since, the energy depletion is generally based on the transmission distance as shown in the section 3.2. Hence, the CHs with the less distance from the BS is preferred in $\mathrm{CH}$ selection and distance from the $\mathrm{CH}$ to $\mathrm{BS}\left(f_{3}\right)$ is expressed in the Eq. (11).

$$
f_{3}=\sum_{i=1}^{M} \operatorname{dis}\left(\mathrm{CH}_{i}, \mathrm{BS}\right)
$$

where, the $\operatorname{dis}\left(\mathrm{CH}_{j}, \mathrm{BS}\right)$ represents the distance from the $j^{\text {th }} \mathrm{CH}$ to BS.

\subsubsection{Node degree}

Node degree represents the amount of cluster members that belongs to the $\mathrm{CH}$. If the $\mathrm{CH}$ has a lesser amount of cluster members, then that $\mathrm{CH}$ sustains for higher duration. Hence the $\mathrm{CH}$ with less node degree is considered and the node degree $\left(f_{4}\right)$ is expressed in the Eq. (12).

$$
f_{4}=\sum_{i=1}^{M} I_{j}
$$




\subsubsection{Node centrality}

Node centrality is defined as the value that classify the nodes based on the distance from the adjacent nodes with the proportion to the network area. This node centrality is considered as 5th objective that is expressed in the following Eq. (13).

$$
f_{5}=\sum_{i=1}^{M} \frac{\sqrt{\left(\sum_{j \epsilon P(i)} \operatorname{dist}^{2}(i, j)\right) / P(i)}}{\text { Area }}
$$

where, the number of nodes present in the clustering range is defined as $P(i)$.

Further, the weighted sum approach is utilized to convert the above stated objectives into single objective as shown in the Eq. (14).

$$
F=\alpha_{1} f_{1}+\alpha_{2} f_{2}+\alpha_{3} f_{3}+\alpha_{4} f_{4}+\alpha_{5} f_{5}
$$

where,

$$
\sum_{i=1}^{5} \alpha_{i}=1, \alpha_{i} \in(0,1)
$$

where, the values of $\alpha_{1}, \alpha_{2}, \alpha_{3}, \alpha_{4}$ and $\alpha_{5}$ are 0.30 , $0.25,0.2,0.15$ and 0.1 respectively. The Eq. (14) is considered as a source of food for the ECMOSSA which is used to update the location of salp as shown in the Eq. (5). Hence, the optimal CHs from the network is selected during the $\mathrm{CH}$ selection process.

\subsection{Cluster formation using potential function}

In cluster formation phase, the non $\mathrm{CHs}$ are allocated to the respective $\mathrm{CHs}$ obtained from the ECMOSSA. The cluster formation mainly considers two different values such as residual energy of the $\mathrm{CH}$ and distance between the nodes to the $\mathrm{CH}$. The cluster formation of the network is obtained using the Eq. (15).

$$
\text { Potential of sensor }\left(N_{i}\right)=\frac{E_{C H}}{\operatorname{dis}\left(N_{i}, C H\right)}
$$

The potential function of sensor is utilized to allocate the non- $\mathrm{CH}$ member to the $\mathrm{CH}$ with higher residual energy and less transmission distance.

The $\mathrm{CH}$ with more residual energy is mainly chosen to minimize the packet drop in the network, because the node with less energy is quickly becoming a dead node. Subsequently, the node with lesser distance to the $\mathrm{CH}$ is considered for minimizing the energy consumption while transmitting the data packets.

\subsection{Route path generation using ECMOSSA}

The routing path is generated by considering the three different fitness function such as residual energy of the $\mathrm{CH}$, distance from the $\mathrm{CH}$ to $\mathrm{BS}$ and node degree. The detailed description is given as follows:

\subsubsection{Salp representation}

In the representation phase, each salp is represented using the route from each $\mathrm{CH}$ to the BS. Similar to the $\mathrm{CH}$ selection, the dimension of each salp in the routing is identical to the amount of the $\mathrm{CHs}$ in the network.

\subsubsection{Salp initialization}

Each salp dimension is equal to the number of the CHs. Consider the $i^{\text {th }}$ salp i.e., $x^{i}=x_{1}^{i}, x_{2}^{i}, \ldots, x_{j}^{i}$, where dimension is represented as $j$ that is similar to the number of CHs, where the location of an each salp is $x_{j}^{i}, 1 \leq a \leq j$ that specifies the next hop $\mathrm{CH}_{\mathrm{j}}$ towards the BS.

\subsubsection{Fitness function formulation for ECMOSSA routing}

In ECMOSSA, there are three different fitness function values such as residual energy $\left(g_{1}\right)$, distance from the $\mathrm{CH}$ to $\mathrm{BS}\left(g_{2}\right)$ and node degree $\left(g_{3}\right)$ are considered to choose an adequate route from the $\mathrm{CH}$ to BS. Then these multiple objectives are changed into a single objective using weighted sum approach as shown in the Eq. (16).

$$
G=\varphi_{1} g_{1}+\varphi_{2} g_{2}+\varphi_{3} g_{3}
$$

where, the $\varphi_{1}, \varphi_{2}$ and $\varphi_{3}$ are the weighted coefficients that is equal to the $0.5,0.3$ and 0.2 respectively. The $g_{1}, g_{2}$ and $g_{3}$ are expressed in the Eqs. (17), (18), and (19) respectively.

$$
\begin{aligned}
& g_{1}=\sum_{i=1}^{M} \frac{1}{E_{C H_{i}}} \\
& g_{2}=\sum_{i=1}^{M} \operatorname{dis}\left(C H_{i}, B S\right) \\
& g_{3}=\sum_{i=1}^{M} I_{j}
\end{aligned}
$$

The $\mathrm{CH}$ with insufficient energy is avoided in the routing path by considering the residual energy as its first priority. The $\mathrm{CH}$ with less residual energy causes the failure of a node in the network. Next, the shortest path is obtained using the distance as second priority 
Table 1. Simulation parameters

\begin{tabular}{|l|l|}
\hline Parameter & Value \\
\hline Number of nodes & 100 \\
\hline Area & $100 \mathrm{~m} \times 100 \mathrm{~m}$ \\
\hline Initial energy & $0.5 \mathrm{~J}$ \\
\hline$E_{\text {elec }}$ & $50 \mathrm{~nJ} / \mathrm{bit}$ \\
\hline$E_{f s}$ & $0.001310 \mathrm{pJ} / \mathrm{bit} / \mathrm{m}^{4}$ \\
\hline$E_{m p}$ & $10 \mathrm{pJ} / \mathrm{bit} / \mathrm{m}^{2}$ \\
\hline Packet length & $4000 \mathrm{bits}$ \\
\hline
\end{tabular}

which helps to decrease the energy consumption. Further, the node degree is taken as $3^{\text {rd }}$ objective to choose the $\mathrm{CH}$ with less non- $\mathrm{CH}$ members. The data transmission is accomplished in the WSN, once the routing path is identified from the $\mathrm{CH}$ to $\mathrm{BS}$.

\section{Experimental results and discussion}

The experimental results and discussion of the ECMOSSA method is described in this section. The implementation and simulation of the ECMOSSA is carried out using the MATLAB R2018a software as well as this software is operated on Windows 7 operating system with Intel core i3 processor and 4GB RAM. In the ECMOSSA, the clustering and routing over the network is accomplished by the SSA with an effective fitness function. Here, 100 sensors are randomly deployed in the area of $100 \mathrm{~m} \times 100 \mathrm{~m}$ to simulate the ECMOSSA. The simulation parameters considered in the ECMOSSA is shown in the following Table 1 .

\subsection{Performance analysis}

The performance of the ECMOSSA is evaluated by means of the alive nodes, total energy consumption, total packets received by the BS, throughput and network lifetime. The ECMOSSA is analysed with three different methods LEACH protocol (i.e., classical algorithm), GWO-DHR [20] and C-SSA [22] with the same specifications mentioned in the Table 1.

\subsubsection{Alive nodes}

Alive nodes are defined as the amount of nodes which has the energy to send the data through the network. Since, a high amount of alive nodes helps to increase the data packets collected by the BS. Fig. 2 shows the alive nodes analysis of the ECMOSSA method with LEACH protocol, GWO-DHR [20] and C-SSA [22]. The alive nodes of the ECMOSSA method is high than the LEACH, GWO-DHR [20] and C-SSA [22]. For example, the LEACH protocol

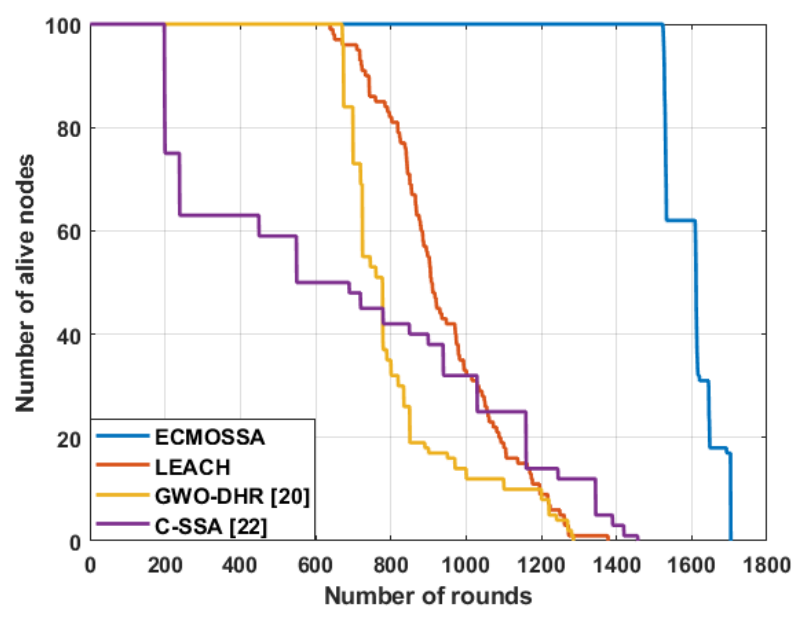

Figure. 2 Performance analysis of alive nodes for ECMOSSA method

obtains less amount of alive nodes, because it uses the single hop transmission during the data transmission.

In this single hop transmission of LEACH, the data packets are directly transmitted to the BS that quickly exhausts the energy of the nodes. But, the ECMOSSA performs the multi hop transmission between the $\mathrm{CH}$ to $\mathrm{BS}$ which used to preserve the energy consumption of the nodes during the data transmission. Hence, the alive nodes of the ECMOSSA method are higher than the LEACH protocol.

\subsubsection{Total energy}

The sensor node dissipates the energy while transmitting and receiving the data packets through the WSN. After dissipation, the sensor node has some remaining energy, which is specified as the total energy of the nodes. The total energy comparison for the ECMOSSA method with LEACH, GWO-DHR [20] and C-SSA [22] is shown in the Fig. 3. From the Fig. 3, it concluded that the ECMOSSA method has higher energy than the LEACH, GWO-DHR [20] and C-SSA [22].

For example, the random selection of $\mathrm{CH}$ in LEACH protocol selects the node in edge of the network as $\mathrm{CH}$ at sometimes. The node's energy consumption is high, when the $\mathrm{CH}$ is located too far from the BS. However, the total energy of the ECMOSSA is improved by considering distance for an optimal $\mathrm{CH}$ selection and by generating an shortest path using the ECMOSSA. This ECMOSSA based $\mathrm{CH}$ and route selection helps to improve the total energy of the nodes. 


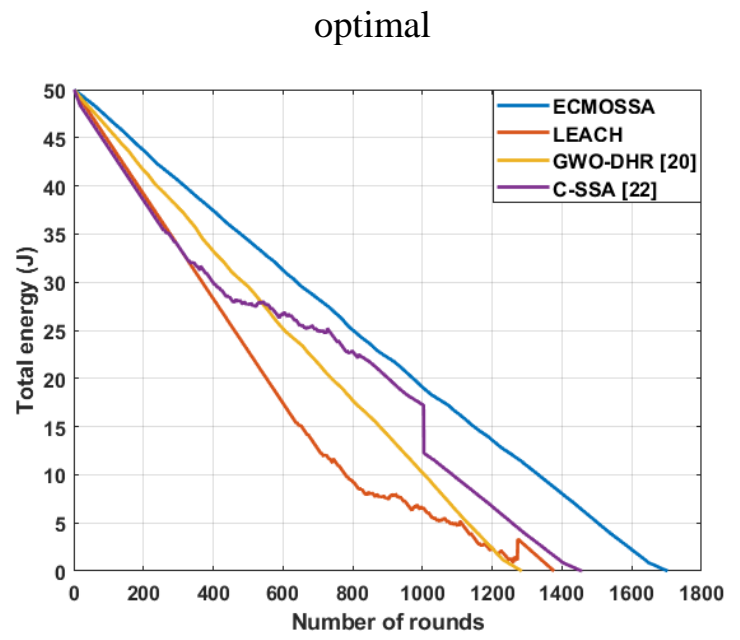

Figure. 3 Performance analysis of total energy for ECMOSSA method

\subsubsection{Total packets received by the BS}

In a network, each sensor sends the data packet to the BS. The amount of packets received by the $\mathrm{BS}$ is mainly depends on the amount of alive nodes and node's residual energy. The number of packets collected by the BS is increased, when both the alive nodes and residual energy provides better results in the WSN. Fig. 4 shows the comparison of total packets received by the BS for ECMOSSA method with LEACH, GWO-DHR [20] and C-SSA [22]. The total packets received by the BS for ECMOSSA method is higher than the LEACH, GWO-DHR [20] and C-SSA [22].

Here, the total packets received by the BS for ECMOSSA method is increased because of high amount of alive nodes and residual energy. Additionally, the residual energy consideration in ECMOSSA routing is used to eliminate the failure node during route generation which helps to increase the data packets received by the BS. Moreover, the

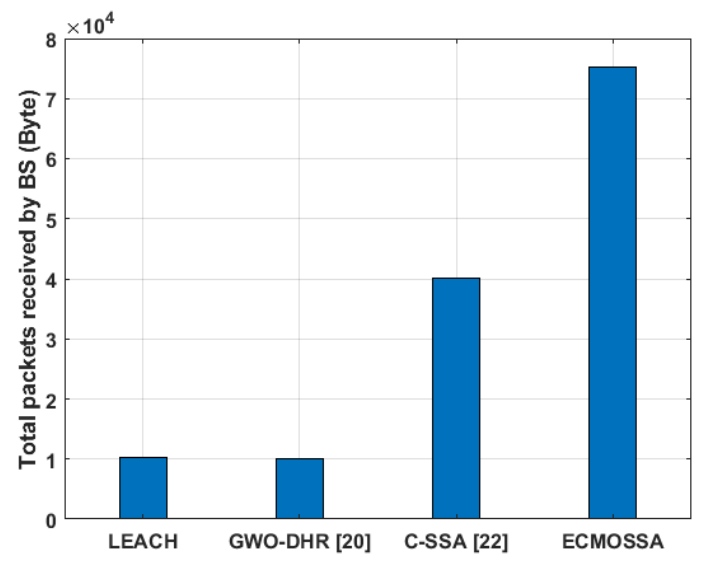

Figure. 4 Performance analysis of total packets received by the BS for ECMOSSA method

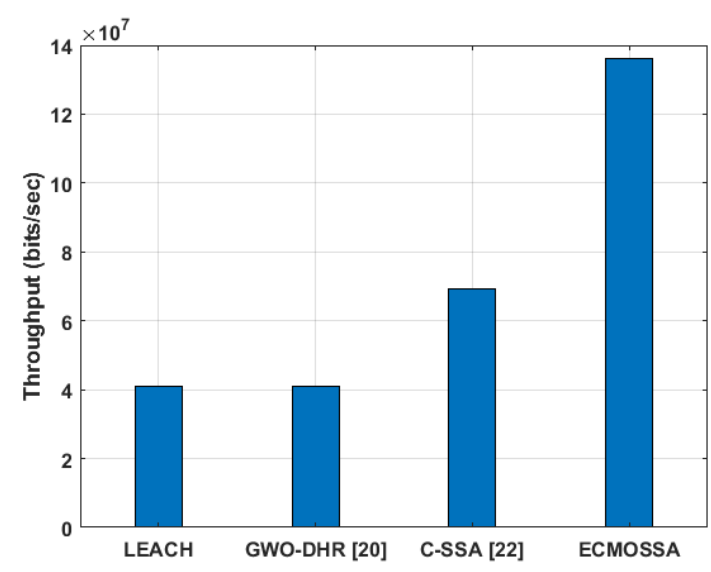

Figure. 5 Performance analysis of throughput for ECMOSSA method

less amount of alive nodes in the LEACH protocol decreases the packets received by the BS.

\subsubsection{Throughput}

Throughput is defined as the number of packets successfully received by the BS and generally it calculated by bits per second.

The throughput comparison for the ECMOSSA method with LEACH, GWO-DHR [20] and C-SSA [22] is shown in the Fig. 5. From the Fig. 5, concluded that the ECMOSSA method has higher throughput when compared to the LEACH, GWODHR [20] and C-SSA [22]. In LEACH protocol, packet loss is occurred through the network, because the remaining energy of the nodes are not considered during the $\mathrm{CH}$ selection process. But, the ECMOSSA used in the clustering and routing process considers the residual energy of the nodes to improve the throughput while transmitting the data packets from the source to the destination.

\subsubsection{Network lifetime}

In WSN, the network lifetime is calculated using 3 metrics such as First Node Die (FND), Half Node Die (HND) and Last Node Die (LND). FND defines the first node which exhausts the energy over the network. Next, the HND defines the $50 \%$ node exhausts their energy in the network. Further, LND defines a $100 \%$ of node dies through the network and this LND used to identify the network becomes inoperative through the WSN.

Fig. 6 shows the comparison of network lifetime for ECMOSSA method with LEACH, GWO-DHR [20] and C-SSA [22]. Here, the network lifetime is analysed in terms of FND, HND and LND. Fig. 6 shows that the FND, HND and LND of the 


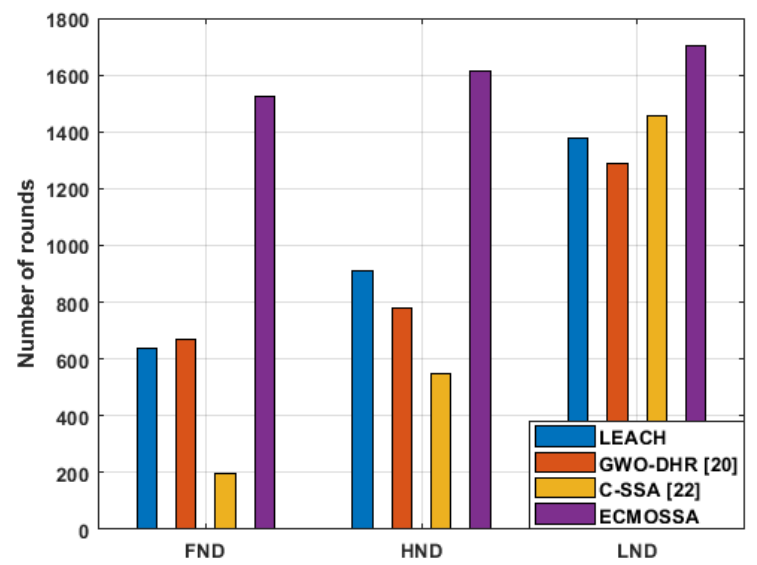

Figure. 6 Performance analysis of network lifetime for ECMOSSA method

ECMOSSA method is high than the LEACH, GWODHR [20] and C-SSA [22]. For example, the LND of the ECMOSSA method is 1704 that is high whencompared to the LND of the LEACH. The lifetime of the LEACH is less due to its inappropriate $\mathrm{CH}$ selection and single hop data transmission. But, in the ECMOSSA method an appropriate fitness function formulation is derived for both the $\mathrm{CH}$ selection and multi hop routing process which leads to increase the lifetime of the network.

\subsection{Comparative analysis}

To justify the effectiveness of the ECMOSSA method, this ECMOSSA is compared with two existing methods such as GWO-DHR [20] and CSSA [22]. In this comparative analysis of ECMOSSA with GWO-DHR [20], 100 sensors are arranged over the network area of $100 \mathrm{~m} \times 100 \mathrm{~m}$. Subsequently, the base station is situated far from the network area i.e., $(150,100)$. From the 100 sensors, a $5 \%$ of nodes are selected as $\mathrm{CH}$ to gather the information from the non- $\mathrm{CH}$ members and the collected information are transmitted to the BS.

Table 2 and Table 3 shows the comparison of the ECMOSSA with GWO-DHR [20] for alive nodes, total energy, throughput and network lifetime. Additionally, the comparison of the ECMOSSA with C-SSA [22] for alive nodes, total energy, throughput and network lifetime are shown in the Table 4 and Table 5. The WSN with 50 sensors are considered to evaluate the performances of ECMOSSA with CSSA [22]. For example, the graphical illustration of lifetime metric for GWO-DHR [20] and ECMOSSA is shown in the Fig. 7.

Table 2. Comparative analysis of the ECMOSSA with GWO-DHR in terms of alive nodes, total energy and throughput

\begin{tabular}{|c|c|c|c|c|c|c|}
\hline \multirow{2}{*}{ Rounds } & \multicolumn{2}{|c|}{ Alive nodes } & \multicolumn{2}{c|}{ Total energy (J) } & \multicolumn{2}{c|}{ Throughput (Mbps) } \\
\cline { 2 - 7 } & GWO-DHR [20] & ECMOSSA & $\begin{array}{c}\text { GWO-DHR } \\
{[\mathbf{2 0}]}\end{array}$ & ECMOSSA & $\begin{array}{c}\text { GWO-DHR } \\
{[\mathbf{2 0}]}\end{array}$ & ECMOSSA \\
\hline 200 & 100 & 100 & 38.48 & 43.70 & 10 & 16 \\
\hline 400 & 100 & 100 & 29.89 & 37.44 & 20 & 32 \\
\hline 600 & 100 & 100 & 21.45 & 31.25 & 31 & 48 \\
\hline 800 & 35 & 100 & 14.57 & 25.02 & 46 & 64 \\
\hline 1000 & 14 & 100 & 10.23 & 19.08 & 60 & 96 \\
\hline 1200 & 10 & 100 & 5.95 & 13.57 & 70 & 96 \\
\hline 1300 & 0 & 100 & 0 & 10.91 & & 89 \\
\hline
\end{tabular}

Table 3. Comparative analysis of the ECMOSSA with GWO-DHR in terms of network lifetime

\begin{tabular}{|c|c|c|}
\hline Lifetime metrics & GWO-DHR [20] & ECMOSSA \\
\hline FND & 671 & 1524 \\
\hline HND & 778 & 1612 \\
\hline LND & 1286 & 1704 \\
\hline
\end{tabular}

Table 4. Comparative analysis of ECMOSSA with C-SSA in terms of alive nodes, total energy and throughputs

\begin{tabular}{|c|c|c|c|c|c|c|}
\hline \multirow{2}{*}{ Rounds } & \multicolumn{2}{|c|}{ Alive nodes } & \multicolumn{2}{c|}{ Total energy (J) } & \multicolumn{2}{c|}{ Throughput (Mbps) } \\
\cline { 2 - 7 } & C-SSA [22] & ECMOSSA & C-SSA [22] & ECMOSSA & C-SSA [22] & ECMOSSA \\
\hline 500 & 47 & 50 & 21.5 & 24.13 & 15 & 20 \\
\hline 1000 & 40 & 50 & 13 & 20.74 & 26 & 40 \\
\hline 1500 & 34 & 50 & 6.5 & 17.33 & 40 & 60 \\
\hline 2000 & 24 & 50 & 2.5 & 13.92 & 53 & 80 \\
\hline
\end{tabular}




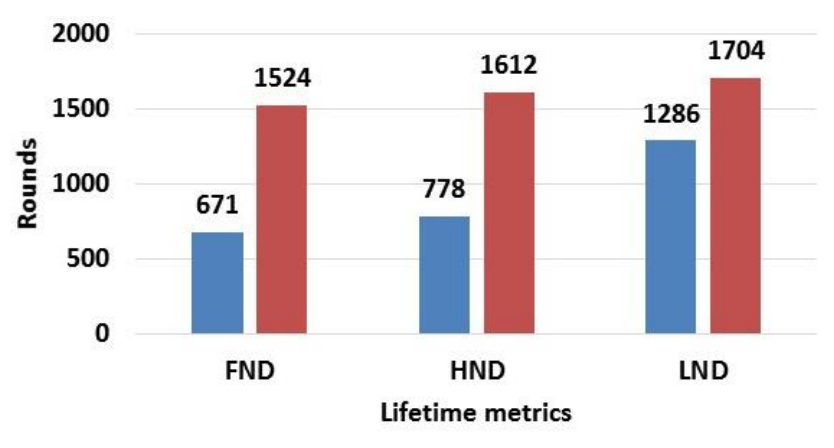

a GWO-DHR [20] $\quad$ ECMOSSA

Figure. 7 Graphical illustration of the network lifetime comparison for ECMOSSA with GWO-DHR

Table 5. Comparative analysis of the ECMOSSA with CSSA in terms of network lifetime

\begin{tabular}{|c|c|c|}
\hline Lifetime metrics & C-SSA [22] & ECMOSSA \\
\hline FND & 250 & 2405 \\
\hline HND & 1995 & 3592 \\
\hline
\end{tabular}

From the comparative analysis, concluded that the ECMOSSA method provides better performance than the GWO-DHR [20] and C-SSA [22]. The GWO-DHR [20] has achieved less performance due to its inappropriate fitness function consideration during the $\mathrm{CH}$ selection. Additionally, the C-SSA [22] provides less performance due to its inappropriate $\mathrm{CH}$ selection using LEACH. In the ECMOSSA clustering, there are five different fitness values such as residual energy, intra cluster distance and distance from the $\mathrm{CH}$ to $\mathrm{BS}$, node degree and node centrality are considered to detect an optimal $\mathrm{CH}$ between the sensors. Next, an appropriate route generation using the ECMOSSA is used for decreasing the energy depletion of the nodes. Accordingly, this ECMOSSA is used to obtain the better network lifetime when compared to the GWODHR [20] and C-SSA [22]. The improved lifetime of the ECMOSSA is results high amount of data packet transmission to the BS.

\section{Conclusion}

In this research paper, the ECMOSSA is used to select an optimal $\mathrm{CHs}$ and generating routing paths through the WSN. There are five different fitness function values such as residual energy, intra cluster distance and distance from the $\mathrm{CH}$ to $\mathrm{BS}$, node degree and node centrality are considered to select the optimal CHs. The selection of $\mathrm{CH}$ with higher residual energy is used to avoid the $\mathrm{CH}$ become dead while transmitting the data in a network. Additionally, the routing path is selected by considering the residual energy of the $\mathrm{CH}$, distance from the $\mathrm{CH}$ to BS and node degree in ECMOSSA. Hence, the shortest path from the ECMOSSA is used to minimize the energy consumption of the nodes that results in better throughput. The results show that the ECMOSSA provides better performance than the both LEACH and GWO-DHR method. The last node dies (i.e., network lifetime) of the ECMOSSA is 1704 that is high when compared to both the LEACH and GWO-DHR method. In future, the performances of the WSN can be improved by using novel optimization algorithms.

\section{Conflicts of Interest}

The authors declare no conflict of interest.

\section{Author Contributions}

The paper conceptualization, methodology, software, validation, formal analysis, investigation, resources, data curation, writing - original draft preparation, writing-review and editing, visualization, have been done by $1^{\text {st }}$ author. The supervision and project administration, have been done by $2^{\text {nd }}$ author.

\section{References}

[1] X. Li, B. Keegan, F. Mtenzi, T. Weise, and M. Tan, "Energy-efficient load balancing ant based routing algorithm for wireless sensor networks", IEEE Access, Vol. 7, pp. 113182-113196, 2019.

[2] H. El Alami and A. Najid, "ECH: An enhanced clustering hierarchy approach to maximize lifetime of wireless sensor networks", IEEE Access, Vol. 7, pp. 107142-107153, 2019.

[3] F. Gao, W. Luo, and X. Ma, "Energy constrained clustering routing method based on particle swarm optimization", Cluster Computing, Vol. 22, No. 3, pp. 7629-7635, 2019.

[4] P. S. Mehra, M. N. Doja, and B. Alam, "Fuzzy based enhanced cluster head selection (FBECS) for WSN", Journal of King Saud UniversityScience, Vol. 32, No. 1, pp. 390-401, 2020.

[5] O. Deepa and J. Suguna, "An optimized QoSbased clustering with multipath routing protocol for wireless sensor networks", Journal of King Saud University-Computer and Information Sciences, Vol. 32, No. 7, pp. 763-774, 2017.

[6] W. X. Xie, Q. Y. Zhang, Z. M. Sun, and F. Zhang, "A clustering routing protocol for WSN based on type-2 fuzzy logic and ant colony optimization", Wireless Personal Communications, Vol. 84, No. 2, pp. 1165-1196, 2015.

[7] P. Nayak and A. Devulapalli, "A fuzzy logicbased clustering algorithm for WSN to extend the 
network lifetime", IEEE sensors Journal, Vol. 16, No. 1, pp. 137-144, 2015.

[8] G. Han and L. Zhang, "WPO-EECRP: Energyefficient clustering routing protocol based on weighting and parameter optimization in WSN", Wireless Personal Communications, Vol. 98, No. 1, pp. 1171-1205, 2018.

[9] R. Vinodhini and C. Gomathy, "MOMHR: A Dynamic Multi-hop Routing Protocol for WSN Using Heuristic Based Multi-objective Function", Wireless Personal Communications, Vol. 111, No. 2, pp. 883-907, 2020.

[10] R. Elhabyan, W. Shi, and M. St-Hilaire, "A Pareto optimization-based approach to clustering and routing in Wireless Sensor Networks", Journal of Network and Computer Applications, Vol. 114, pp. 57-69, 2018.

[11] N. Mahesh and S. Vijayachitra, "DECSA: hybrid dolphin echolocation and crow search optimization for cluster-based energy-aware routing in WSN", Neural Computing and Applications, Vol. 31, No. 1, pp. 47-62, 2018.

[12] S. A. Sert, A. Alchihabi, and A. Yazici, "A twotier distributed fuzzy logic based protocol for efficient data aggregation in multihop wireless sensor networks", IEEE Transactions on Fuzzy Systems, Vol. 26, No. 6, pp. 3615-3629, 2018.

[13] M. B. Shyjith, C. P. Maheswaran, and V. K. Reshma, "Optimized and Dynamic Selection of Cluster Head Using Energy Efficient Routing Protocol in WSN", Wireless Personal Communications, pp. 1-23, 2020.

[14] J. Yang and F. Liu, "Greedy discrete particle swarm optimization based routing protocol for cluster-based wireless sensor networks", Journal of Ambient Intelligence and Humanized Computing, pp. 1-16, 2017.

[15] S. Kaur and R. Mahajan, "Hybrid meta-heuristic optimization based energy efficient protocol for wireless sensor networks", Egyptian Informatics Journal, Vol. 19, No. 3, pp. 145-150, 2018.

[16] V. K. Chawra and G. P. Gupta, "Salp: metaheuristic-based clustering for wireless sensor networks", In: Proc. of Nature-Inspired Computing Applications in Advanced Communication Networks, IGI Global, pp. 41-56, 2020.

[17] P. Lalwani, H. Banka, and C. Kumar, "CRWO: Clustering and routing in wireless sensor networks using optics inspired optimization", Peer-to-Peer Networking and Applications, Vol. 10, No. 3, pp. 453-471, 2017.

[18] N. Mittal, U. Singh, and B. S. Sohi, "An energyaware cluster-based stable protocol for wireless sensor networks", Neural Computing and Applications, Vol. 31, No. 11, pp. 7269-86, 2017.

[19] P. Lalwani, H. Banka, and C. Kumar, "BERA: a biogeography-based energy saving routing architecture for wireless sensor networks", Soft Computing, Vol. 22, No. 5, pp. 1651-1667, 2018.

[20] S. M. H. Daneshvar, P. A. A. Mohajer, and S. M. Mazinani, "Energy-efficient routing in WSN: A centralized cluster-based approach via grey wolf optimizer", IEEE Access, Vol. 7, pp. 170019170031, 2019.

[21] N.A. Morsy, E. H. AbdelHay, and S. S. Kishk, "Proposed Energy Efficient Algorithm for Clustering and Routing in WSN", Wireless Personal Communications, Vol. 103, No. 3, pp. 2575-2598, 2018.

[22] A. Vinitha, M. S. S. Rukmini, and D. Sunehra, "Energy-efficient multihop routing in WSN using the hybrid optimization algorithm", International Journal of Communication Systems, Vol. 33, No. 12, pp. e4440, 2020.

[23] S. Mirjalili, A. H. Gandomi, S. Z. Mirjalili, S. Saremi, H. Faris, and S. M. Mirjalili, "Salp Swarm Algorithm: A bio-inspired optimizer for engineering design problems", Advances in Engineering Software, Vol. 114, pp. 163-191, 2017. 